
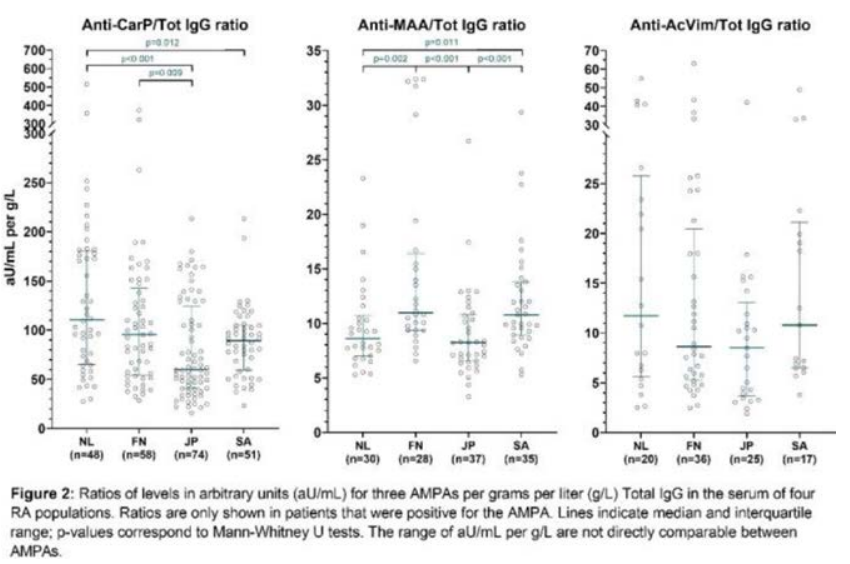

RA populations. Ratios are only shown in patients that were positive for the AMPA. Lines indicate median and interquartile range; p-vat
AMPAs

Disclosure of Interests: Emma C. de Moel: None declared, Veerle Derksen: None declared, Leendert A Trouw: None declared, Chikashi Terao: None declared, Mohammed Tikly: None declared, Hani El-Gabalawy: None declared, Holger Bang Grant/research support from: Employee of Orgentec Diagnostika, Thomas Huizinga Grant/research support from: Ablynx, Bristol-Myers Squibb, Roche, Sanofi, Consultant of: Ablynx, Bristol-Myers Squibb, Roche, Sanofi, Rene Toes: None declared, Diane van der Woude: None declared

DOI: 10.1136/annrheumdis-2020-eular.3146

\section{SAT0586 PREVALENCE AND RISK FACTORS FOR CARDIO- METABOLIC ABNORMALITIES IN PATIENTS WITH INFLAMMATORY ARTHRITIS ATTENDING CARDIO- RHEUMATOLOGY PRIMARY PREVENTION CLINICS}

L. Eder ${ }^{1}$, S. Akhtari ${ }^{1}$, P. Harvey ${ }^{1}$, K. Bindee ${ }^{2} .{ }^{~}$ Women's College Hospital, University of Toronto, Toronto, Canada; ${ }^{2}$ Sinai Health System, University of Toronto, Toronto, Canada

Background: Cardio-metabolic abnormalities are common in patients with inflammatory arthritis (IA) but tend to be under-recognized and under-treated. Objectives: We aimed to compare the prevalence and risk factors for cardio-metabolic abnormalities between patients with rheumatoid arthritis (RA), psoriatic arthritis (PsA) and ankylosing spondylitis (AS).

Methods: Consecutive patients enrolled in the University of Toronto Cardio-Rheumatology Network from July 2017 to August 2019 were analyzed. This is a primary prevention program that uses structured clinical, laboratory and multimodal imaging to diagnose and treat cardiovascular disease (CVD). Patients with a rheumatologist-confirmed diagnosis of RA, PsA or AS with no known CVD were evaluated. Information about IA diagnosis, medications and comorbidities was recorded. Each patient was evaluated by a cardiologist focusing on CVD risk assessment. We evaluated the prevalence of previously recorded and newly recognized cardio-metabolic risk factors including hypertension, dyslipidemia, obesity and diabetes. The prevalence of these abnormalities was compared between IA diagnoses. Regression models were used to assess the association between diagnosis and cardio-metabolic abnormalities after adjusting for demographics, smoking, BMI, measures of disease activity and medications.

Results: A total of 358 patients (201 RA, 124 PsA, 33 AS) were assessed (mean age $59 \pm 10.5$ years, $68.7 \%$ female). Hypertension was reported in $33 \%$, dyslipidemia in $26.8 \%$, diabetes mellitus in $8.9 \%$ and overweight/obesity in $69.7 \%$ (Figure 1). Newly detected elevations in lipids were frequent for triglycerides (9.3\%), non-HDL-cholesterol (6\%,) and LDL-cholesterol (2.7\%). Elevated HbA1c occurred in $1.4 \%$ and newly diagnosed hypertension occurred in $9.8 \%$. A total of $32.8 \%$ patients required a change or initiation of medications for their cardio-metabolic abnormalities (21.7\% lipid-lowering therapy, $14.6 \%$ aspirin, $11.1 \%$ anti-hypertension therapy). Patients with PsA had the highest prevalence of cardio-metabolic abnormalities including dyslipidemia, obesity and hypertension. Having hypertension (prior or new diagnosis), elevated levels of triglycerides, non-HDL cholesterol, total cholesterol and BMI were associated with PsA vs. RA after adjusting for potential confounders (all $\mathrm{p}<0.05$ ) (Figure 2). No significant association was found between cardio-metabolic abnormalities and AS vs. PsA or RA.

Conclusion: Dedicated cardio-rheumatology clinics have improved CVD screening and management in an IA population. The burden of cardio-metabolic abnormalities is elevated in PSA and suggests that tailored strategies to reduce adverse CVD events are particularly needed in this subgroup.
Figure 1 - The Prevalence of Cardiovascular Risk Factors (Previously diagnosed and Newly diagnosed)
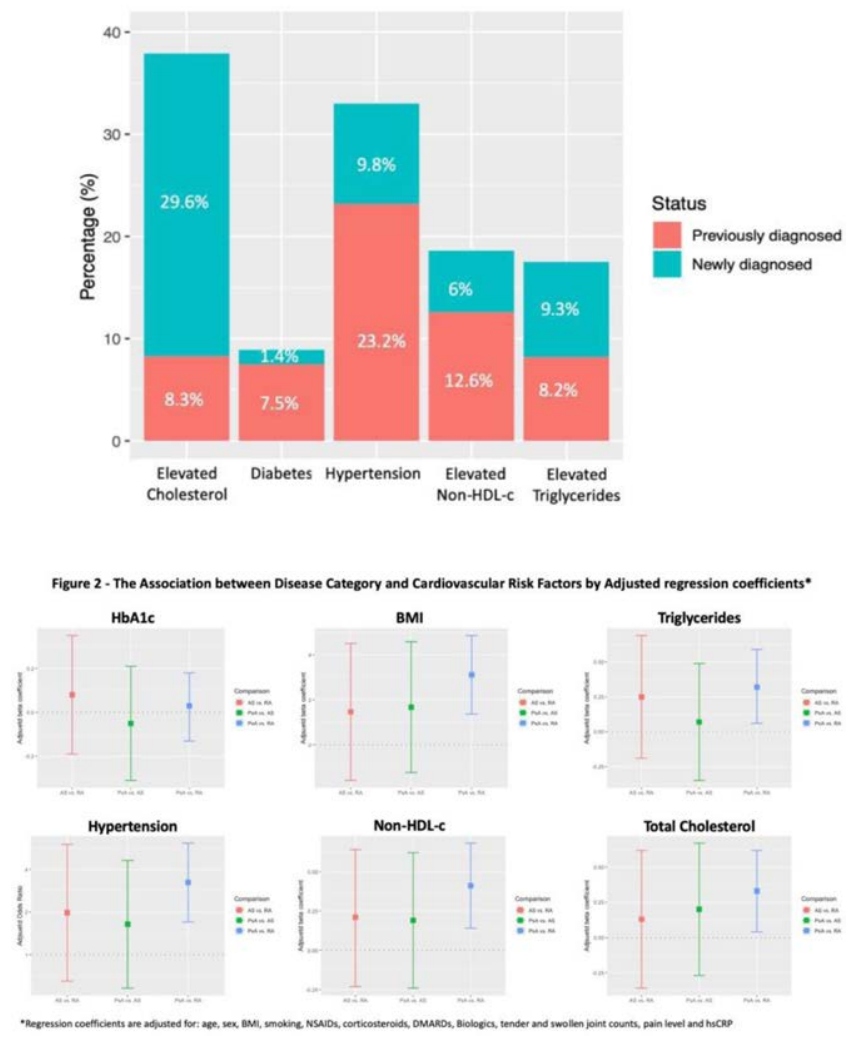

Disclosure of Interests: Lihi Eder Grant/research support from: Abbvie, Lily, Janssen, Amgen, Novartis, Consultant of: Janssen, Speakers bureau: Abbvie, Lily, Janssen, Amgen, Novartis, Shadi Akhtari: None declared, Paula Harvey: None declared, Kuriya Bindee Grant/research support from: Abbvie, Pfizer, Sanofi, BMS, Consultant of: Abbvie, Eli Lily, Pfizer DOI: 10.1136/annrheumdis-2020-eular.1228

\section{SAT0587 MACHINE-LEARNING DERIVED ALGORITHMS FOR OUTCOMES PREDICTION IN RHEUMATIC DISEASES: APPLICATION TO RADIOGRAPHIC PROGRESSION IN EARLY AXIAL SPONDYLOARTHRITIS}

R. Garofoli ${ }^{1}$, M. Resche-Rigon ${ }^{2}$, M. Dougados ${ }^{1}$, D. Van der Heijde ${ }^{3}$, C. Roux ${ }^{1}$, A. Moltó ${ }^{1}{ }^{1}$ Hospital Cochin, Rheumatology, Paris, France; ${ }^{2}$ Hospital Saint-Louis Ap-Hp, Paris, France; ${ }^{3}$ Leiden University Medical Center, Leiden, Netherlands

Background: Axial spondyloarthritis (axSpA) is a chronic rheumatic disease that encompasses various clinical presentations: inflammatory chronic back pain, peripheral manifestations and extra-articular manifestations. The current nomenclature divides axSpA in radiographic (in the presence of radiographic sacroilitis) and non-radiographic (in the absence of radiographic sacroiliitis, with or without MRI sacroiliitis. Given that the functional burden of the disease appears to be greater in patients with radiographic forms, it seems crucial to be able to predict which patients will be more likely to develop structural damage over time. Predictive factors for radiographic progression in axSpA have been identified through use of traditional statistical models like logistic regression. However, these models present some limitations. In order to overcome these limitations and to improve the predictive performance, machine learning (ML) methods have been developed.

Objectives: To compare ML models to traditional models to predict radiographic progression in patients with early axSpA.

Methods: Study design: prospective French multicentric cohort study (DESIR cohort) with 5years of follow-up. Patients: all patients included in the cohort, i.e. 708 patients with inflammatory back pain for $>3$ months but $<3$ years, highly suggestive of axSpA. Data on the first 5 years of follow-up was used. Statistical analyses: radiographic progression was defined as progression either at the spine (increase of at least 1 point per 2 years of mSASSS scores) or at the sacroiliac joint (worsening of at least one grade of the mNY score between 2 visits). Traditional modelling: we first performed a bivariate analysis between our outcome (radiographic progression) and explanatory variables at baseline to select the variables to be included in our models and then built a logistic regression model (M1). Variable selection for traditional models was performed with 2 different methods: stepwise selection based on 